\title{
Expression of bronchodilator response using forced oscillation technique measurements: absolute
} versus relative

\section{To the Editors:}

We refer to the recent study by OOSTVEen et al. [1] on bronchodilator response in 4-yr-old children with different wheezing phenotypes. The forced oscillation technique is particularly suited to 2-7-yr-old children, and its suitability to assess bronchodilator responsiveness is still being investigated. Thus, the study is of great clinical relevance and, though of a narrow age range, is a welcome step forward from previous studies characterising bronchodilator responses assessed using measures of airway resistance in preschool children [2-4]. However, we would like to raise a point we feel to be of importance.

The authors argued for the case of using the absolute change pre- and post-bronchodilator to express a bronchodilator response in children assessed using forced oscillation technique (FOT) variables. They reasoned that expressing the response as a relative change from baseline homogenises the different responses of subjects with different baseline bronchomotor tone, reflected by their different baseline lung function values.

The reason we advocate the use of the relative over the absolute response assessed using airway resistance from oscillometric methods is that the absolute response is generally dependent on lung function at baseline [2, 4]. Statistically, assessment of magnitude of a treatment effect should be adjusted for any differences between the groups or variability in the pretreatment values [5]. For instance, for the forced expiratory volume in $1 \mathrm{~s}$ (FEV1) it has been pointed out that since the absolute change between repeated measurements is constant across patients with varying FEV1 levels, it is the absolute change that should be used when expressing any treatment differences [6]. More accurately, expressing the bronchodilator response using the absolute change is valid provided this change is independent of the baseline value, otherwise the relative change should be used [7]. Dependence on age or height can be accounted for using \% predicted values. Note that expressing the bronchodilator response in terms of absolute changes in z-scores is statistically identical to using absolute changes when the data are homoscedastic, though it may still be useful to use z-scores when lung function is already expressed in this manner or if the data are heteroscedastic.

In more physiological terms, for airway resistance measures, the smaller the airway calibre (hence the larger the baseline resistance), the more profound the effect of any increases to airway calibre. Thus, the same change in airway calibre represents a larger effective change in a small airway than in a large airway. Bronchomotor tone should be reflected by changes to airway calibre upon the administration of a bronchodilator, rather than by baseline lung function per se. A larger baseline bronchomotor tone would thus result in a larger bronchodilator response and represents our signal of interest, but this signal needs to normalised by the effect of a smaller initial airway calibre. Therefore, we would argue that while expressing the response in terms of relative changes "homogenises" the difference between subjects, it is a difference attributable to a factor that is not the main effect of interest but rather can confound it, and therefore needs to be corrected for.

Until more data on how to express a bronchodilator response assessed using airway resistance become available, we would recommend that authors of individual studies first ascertain whether the change with bronchodilator is related to factors such as age, height and baseline lung function. If yes, then these factors should be taken into account, especially when subjects of a wide range of ages, height or baseline lung function are studied.

\section{Thamrin*, C.L. Gangell ${ }^{\#}$, M.M.H. Kusel ${ }^{\#}$, A. Schultz ${ }^{\text {, }}$ G.L. Hall", , S.M. Stick ${ }^{\star}$ and P.D. Sly ${ }^{\#}$}

*Division of Respiratory Medicine, Dept of Paediatrics, Inselspital and University of Bern, Switzerland. ${ }^{\text {TTelethon }}$ Institute for Child Health Research, Centre for Child Health Research, University of Western Australia, and "Respiratory Medicine, Princess Margaret Hospital for Children, Perth, Australia.

Correspondence: C. Thamrin, Division of Paediatric Respiratory Medicine, University Children's Hospital of Bern, Inselspital, 3010 Bern, Switzerland. E-mail: cindy.thamrin@insel.ch

Statement of Interest: None declared.

\section{REFERENCES}

1 Oostveen E, Dom S, Desager $\mathrm{K}$, et al. Lung function and bronchodilator response in 4-year-old children with different wheezing phenotypes. Eur Respir J 2010; 35: 865-872.

2 Hellinckx J, De Boeck K, Bande-Knops J, et al. Bronchodilator response in 3-6.5 years old healthy and stable asthmatic children. Eur Respir J 1998; 12: 438-443. 
3 Nielsen KG, Bisgaard H. Discriminative capacity of bronchodilator response measured with three different lung function techniques in asthmatic and healthy children aged 2 to 5 years. Am J Respir Crit Care Med 2001; 164: 554-559.

4 Thamrin C, Gangell CL, Udomittipong K, et al. Assessment of bronchodilator responsiveness in preschool children using forced oscillations. Thorax 2007; 62: 814-819.

5 Altman DG, Doré CJ. Randomisation and baseline comparisons in clinical trials. Lancet 1990; 335: 149-153.

6 Vale JR, Gulsvik A, Kongerud J. Random error with the FEV1 = case for absolute values. Lancet 1981; 2: 313.

7 Cotes JE. Absolute FEV1 values. Lancet 1981; 2: 423.

DOI: $10.1183 / 09031936.00025310$

\section{From the authors:}

We thank C. Thamrin and co-workers for their interest in our paper and their reflection, which continues the discussion on how to express bronchodilator responsiveness. This has been an important topic of debate for decades in the adult and the paediatric pulmonary field. In their letter, C. Thamrin and coworkers state that if a bronchodilator change is related to anthropometric factors or baseline lung function, these factors should be taken into account.

The worse the baseline lung function the more room for improvement, at least for the asthmatic patient. This has been recognised and translated in the guideline that the bronchodilator response as assessed with forced expiration is expressed as percent change from baseline for forced expiratory volume in $1 \mathrm{~s}$ (FEV1) [1, 2]. Of course, in order to be significant, the response must exceed the threshold for natural short-term variability and, therefore, the response in FEV1 should exceed $200 \mathrm{~mL}$. The 95th percentile for the increase in FEV1 in healthy adults is $\sim 10 \%$ baseline [1]. Asthma is characterised by episodic shortness of breath and variable airway obstruction. A reversibility in FEV1 after bronchodilation $\geqslant 12 \%$ and $\geqslant 200 \mathrm{~mL}$ indicates a diagnosis of asthma in children and adults according to the 2009 Global Initiative for Asthma guidelines [2].

The worse the baseline lung function the more room for improvement. However, contrary to FEV1, which decreases with increasing level of airway obstruction, airway resistance increases when lung function worsens. By expressing the bronchodilator response as percent change from the baseline airway resistance, the differences that existed between groups with different baseline lung function and disappear after bronchodilation will be blunted. This is exactly what we found in our study [3]. We measured baseline lung function and bronchodilator response in 4-yrold children who took part in a prospective birth cohort study in Antwerp, Belgium. The group of children with persistent wheeze had significantly larger baseline resistance and a significantly larger absolute change in resistance after bronchodilation than the group of children who never wheeze. However, the relative changes in the two groups of children were similar (table 1 in the online supplement to our manuscript [3]). Similarly, when we used the 95th percentile of the absolute bronchodilator change in resistance, significantly more responders were found in the group of children with persistent wheeze compared with the group with never wheeze (13\% and $4 \%$, respectively). No significant differences in responders were found in the two groups when using a threshold based on the relative change (table 3 in our manuscript [3]).

It has been documented that the bronchodilator response depends on anthropometric variables and baseline lung function. Both for the relative change in FEV1 as assessed in adults and the absolute change in resistance assessed in (pre)school children, multiple studies have revealed these dependencies [4-7]. The real challenge is to find the threshold value in the expression of the bronchodilator response which best separates the asthmatic from the healthy response, especially in the pre-school child who is unable to perform forced expiration reliably. Although our study was not designed to find an answer to this question, the results strongly suggest that expression of the response as absolute change should not be ruled out.

\section{E. Oostveen*, S. Dom ${ }^{\#}$, K. Desager ${ }^{\top}$, M. Hagendorens", W. De Backer* and J. Weyler ${ }^{\#}$}

Depts of *Pulmonary Medicine, "Epidemiology and Social Medicine, and "Paediatrics, Antwerp University Hospital and University of Antwerp, Antwerp, Belgium.

Correspondence: E. Oostveen, Dept of Pulmonary Medicine, Antwerp University Hospital, 2650 Antwerp-Edegem, Belgium. E-mail: ellie.oostveen@uza.be

Statement of Interest: None declared.

\section{REFERENCES}

1 Pellegrino R, Viegi G, Brusasco V, et al. Interpretative stategies for lung function tests. Eur Respir J 2005; 26: 948-968.

2 Global Initiative for Asthma. Global Strategy for Asthma Management and Prevention: Updated 2009 www.ginasthma.org/ guidlineitem.asp? $11=2 \& 12=1 \&$ intId =1561 Date last accessed: March 1, 2010.

3 Oostveen E, Dom S, Desager K, et al. Lung function and bronchodilator response in 4-year-old children with different wheezing phenotypes. Eur Respir J 2010; 35: 865-872.

4 Weir DC, Sherwood Burge P. Measures of reversibility in response to bronchodilators in chronic airflow obstruction: relation to airway calibre. Thorax 1991; 46: 43-46.

5 Brand PLP, Baraldi E, Bisgaard H, et al. Definition, assessment and treatment of wheezing disorders in preschool children: an evidencebased approach. Eur Respir J 2008; 32: 1096-1110.

6 Thamrin C, Gangell CL, Udomittipong K, et al. Assessment of bronchodilator responsiveness in preschool children using forced oscillations. Thorax 2007; 62: 814-819.

7 Nielsen KG, Bisgaard H. Discriminative capacity of bronchodilator response measured with three different lung function techniques in asthmatic and healthy children aged 2 to 5 years. Am J Respir Crit Care Med 2001; 164: 554-559. 